\title{
ESTUDO DO PROCESSO SACARIFICAÇÃO E FERMENTAÇÃO SIMULTÂNEA (SFS) EM BIORREATOR EM BATELADA ALIMENTADA VISANDO A REDUÇÃO DE CUSTOS DA PRODUÇÃO DE ETANOL A PARTIR DE BAGAÇO DE CANA-DE-AÇÚCAR
}

\author{
A. DE A. GUILHERME ${ }^{1}$, L. P. DE SOUZA ${ }^{1}$, P. V. F. DANTAS ${ }^{1}$, E. S. DOS SANTOS ${ }^{1}$, F. A. \\ N. FERNANDES ${ }^{2}$, G. R. DE MACEDO ${ }^{1}$ \\ ${ }^{1}$ Universidade Federal do Rio Grande do Norte - Departamento de Engenharia Química \\ ${ }^{2}$ Universidade Federal do Ceará - Departamento de Engenharia Química \\ Contato: alexandredearaujoguilherme@gmail.com
}

RESUMO: Visando a demanda por energia de forma renovável, este trabalho teve como objetivo o estudo da produção de etanol a partir de bagaço de cana-de-açúcar pelo processo SFS em sistema batelada alimentada. A levedura Saccharomyces cerevisiae PE-2 foi utilizada como agente de fermentação na quantidade de $1,0 \mathrm{~g} / \mathrm{L}$ de inoculo e a enzima usada foi a NS22086 (Novozymes) com atividade inicial de 15 FPU/g de celulose. O bagaço foi prétratado com ácido sulfúrico em uma primeira etapa e hidróxido de sódio em uma segunda etapa. Os ensaios em biorreator foram realizados em dorna de $2 \mathrm{~L}$ com $1 \mathrm{~L}$ de volume reacional fazendo-se alimentação com $6 \%$ de celulose do bagaço de cana em 3 etapas. Desta forma, foi possível produzir etanol com rendimentos próximos de $90 \%$ trabalhando-se com menor quantidade de enzima em um sistema com melhor transferência de massa e menor demanda de potência para agitação quando comparado ao processo em batelada.

\section{INTRODUÇÃO}

O Setor energético está enfrentando uma incerteza sem precedentes em todo o mundo. A crise econômica mundial de 2008/2009 deixou o mercado de energia em turbulência e o ritmo em que a economia global se recupera detém a chave para as perspectivas de energias para os próximos anos. Em resposta a esta questão, esforços contínuos têm sido feitos na exploração de fontes alternativas de energias renováveis para o desenvolvimento sustentável (Zhang et al., 2013).

A biomassa vegetal, rica em material lignocelulósico, está sendo estudada no mundo todo como uma fonte renovável de carbono e energia. Sem dúvida alguma, o etanol é o mais importante produto final obtido pela fermentação de hidrolisados lignocelulósicos, porém outros produtos também podem ser obtidos. Xilitol e lipídeos podem ser produzidos por 
processos fermentativos a partir de xilose. Ácidos orgânicos, antibióticos, aromas e uma gama de outros produtos podem ser produzidos a partir da glicose através de bioprocessos.

Para a produção de etanol de segunda geração (etanol celulósico) é preciso que se obtenham monômeros de carboidratos como a glicose, proveniente da estrutura da parede celular vegetal composta principalmente de lignocelulose. Desta forma, diferentemente do processo convencional, para a produção de etanol de segunda geração é preciso que o material lignocelulósico seja submetido a um pré-tratamento químico, físico, biológico ou combinado e a uma hidrólise enzimática para que se tenha substrato disponível para a fermentação alcoólica.

Os pré-tratamentos são utilizados para se aumentar a conversão do material lignocelulósico em açúcares redutores como celobiose e glicose (Sun e Cheng, 2002) através do processo de hidrólise enzimática. Eles atuam desestruturando a matriz lignocelulósica, reduzindo as quantidades de lignina e hemicelulose e modificando a estrutura cristalina da celulose de forma a deixá-la mais susceptível ao ataque enzimático (Silverstein et al., 2007).

Pré-tratamentos ácidos envolvendo ácido sulfúrico, nítrico ou clorídrico podem solubilizar parte da hemicelulose expondo ainda mais a celulose ao ataque enzimático (Schell et al., 2003). Pré-tratamentos alcalinos se referem ao uso de soluções alcalinas como hidróxido de sódio, dentre outras, usadas basicamente para remover lignina e diminuir o grau de cristalinidade da celulose (Chang e Holtzapple, 2000).

A celulose pode ser hidrolisada, por enzimas celulases, em glicose, e esta, pode ser fermentado para produzir etanol (Yang et al., 2011) em um processo com duas etapas. Entretanto, estes dois processos podem ser realizados em uma só etapa denominada de sacarificação e fermentação simultânea (SFS) (Vasquez et al., 2007) objetivando redução de tempo e energia diminuído custos de processo.

Este trabalho teve como objetivo um estudo cinético em biorreator do processo SFS em processo batelada alimentada visando à redução de custos reduzindo a quantidade de enzimas utilizadas no processo e melhorando a reologia do sistema, assim, reduzindo gastos com demanda de potência no processo de agitação do reator.

\section{MATERIAL E MÉTODOS}

\subsection{Enzimas, levedura e bagaço de cana-de-açúcar}

As enzimas utilizadas neste trabalho foram (NS22086) (220 FPU/mL e $4500 \mathrm{CBU} / \mathrm{mL}$ ) que corresponde às celulases cedidas pela Novozymes (Bagsvaerd, Dinamarca). A atividade enzimática foi determinada segundo Ghose (1987) em FPase (Atividades de celulases medida através da determinação da concentração de açúcares redutores liberados durante a degradação de uma fita de papel de filtro) e Celobiase (Atividades de celobiases medida através da determinação da concentração de glicose liberada durante a degradação de celobiose). O substrato utilizado foi o bagaço de cana-de-açúcar cedido gentilmente pela 
Usina Estivas (Arês - RN, Brasil), e a levedura utilizada foi uma linhagem capaz de produzir etanol a partir de glicose, a Saccharomyces cerevisiae PE- 2 (Basso et al., 2008).

\subsection{Estoque de leveduras, pré-inoculo e inoculo}

A levedura foi mantida, durante os ensaios a $-20{ }^{\circ} \mathrm{C}$ em glicerol estéril sendo o estoque renovado a cada seis meses segundo Silva et al., (2008). Também foi mantida em meio YEPD inclinado a $4{ }^{\circ} \mathrm{C}$ sendo este estoque renovado a cada dois meses.

Para a produção do pré-inoculo e inoculo foi utilizado o meio de cultura nas seguintes proporções; glicose $(30,0 \mathrm{~g} / \mathrm{L})$, extrato de levedura $(5,0 \mathrm{~g} / \mathrm{L}),\left(\mathrm{NH}_{4}\right)_{2} \mathrm{SO}_{4}(10,0 \mathrm{~g} / \mathrm{L}), \mathrm{KH}_{2} \mathrm{PO}_{4}$ $(4,5 \mathrm{~g} / \mathrm{L}), \mathrm{MgSO}_{4} .7 \mathrm{H}_{2} \mathrm{O}(1,0 \mathrm{~g} / \mathrm{L})$ e $\mathrm{ZnSO}_{4}(0,65 \mathrm{~g} / \mathrm{L})$.

Para o preparo do pré-inoculo foi utilizado erlenmyers de $250 \mathrm{~mL}$ com $100 \mathrm{~mL}$ de meio de cultura, velocidade de agitação de $150 \mathrm{RPM}$, temperatura de $30{ }^{\circ} \mathrm{C}$. Uma colônia da levedura, crescida em placa de Petri, foi transferida para os frascos de erlenmyers onde foi incubada por 23 horas.

A partir do pré-inoculo, foi transferido $5 \mathrm{~mL}$ da suspensão celular para os meios de inoculo. As condições de processos foram iguais às do pré-inoculo com exceção da temperatura que foi de $35^{\circ} \mathrm{C}$ e o tempo de incubação que foi de 10 horas.

\subsection{Pré-tratamento no bagaço de cana}

O pré-tratamento ácido alcalino foi realizado utilizando bagaço na quantidade de sólidos de $20 \%$ (p/v) imerso em uma solução de ácido sulfúrico 2\% (v/v) submetido a uma temperatura de $121^{\circ} \mathrm{C}$ por 30 minutos, segundo Guo et al., (2009). Em seguida, a fração sólida foi lavada com água até o valor do $\mathrm{pH}$ ficar próximo ao $\mathrm{pH}$ da água de lavagem. Após o pré-tratamento ácido, o material foi submetido a um pré-tratamento alcalino para a deslignificação onde foi utilizada uma quantidade de $20 \%(\mathrm{p} / \mathrm{v})$ do material sólido submerso em solução de hidróxido de sódio $4 \%(\mathrm{p} / \mathrm{v})$ submetido a uma temperatura de $121^{\circ} \mathrm{C}$ por 30 minutos, segundo Vasquez et al., (2007). Em seguida, o pH foi corrigido para 7,0 utilizando ácido clorídrico para posterior lavagem. O pré-tratamento visa à remoção de parte da hemicelulose (Schell et al., 2003), lignina e redução do grau de cristalinidade da celulose (Chang e Holtzapple, 2000).

\subsection{Cinética em biorreator}

O biorreator utilizado nesta etapa foi uma adaptação de uma dorna de 2 L (Applikon Dependable Instruments, Holanda) com controle de agitação (mod P100/ADI 1032, Applikon, Holanda) acoplados a uma torre de controle de $\mathrm{pH}$, nível e nutriente (mod TEC BIO, Tecnal, 
São Paulo/Brasil) usando um banho termostático para controle de temperatura (mod TE 184, Tecnal, São Paulo/Brasil).

O estudo cinético foi realizado com $1 \mathrm{~L}$ de volume reacional. As quantidades foram de 1,0 g/L de inoculo, a atividade enzimática inicial foi de $15 \mathrm{FPU} / \mathrm{g}$ de celulose e a quantidade de celulose foi de $6 \%$ contida no bagaço de cana de acordo com resultados de estudos anteriores. $\mathrm{O}$ meio de cultura foi; extrato de levedura $(4,0 \mathrm{~g} / \mathrm{L}),\left(\mathrm{NH}_{4}\right)_{2} \mathrm{SO}_{4}(2,0 \mathrm{~g} / \mathrm{L}), \mathrm{KH}_{2} \mathrm{PO}_{4}$ $(2,0 \mathrm{~g} / \mathrm{L}), \mathrm{MgSO}_{4} \cdot 7 \mathrm{H}_{2} \mathrm{O}(0,75 \mathrm{~g} / \mathrm{L})$ e a celulose contida no bagaço de cana como fonte de carbono e energia.

Um primeiro ensaio foi realizado, durante 12 horas, apenas com $2 \%$ de celulose contida no bagaço de cana chamado (CBA-A) para se calcular o final de fase Log e estimar a taxa de consumo de celulose em (g/L.h) ao final de fase Log. Os resultados deste ensaio serviu para determinar a segunda e terceira alimentação no ensaio completo descrito a seguir.

Um estudo cinético completo foi feito (CBA-B) com $6 \%$ de celulose alimentada em três vezes em tempos determinados. A segunda alimentação foi realizada no final da fase Log para se evitar um estresse no micro-organismo causado por falta de substrato. A terceira alimentação foi feita usando a estimativa do consumo de celulose em (g/L.h). O crescimento celular foi monitorado após a segunda alimentação, desta forma, seria possível calcular uma nova fase exponencial para se estimar um tempo mais adequado de se fazer a terceira alimentação caso um terceiro estudo cinético fosse preciso.

Amostras foram coletadas em intervalos de tempo determinados para análises de açúcares celobiose, glicose, xilose e celulose, bem como, a produção de etanol e glicerol, se fazer o monitoramento do crescimento microbiano e da atividade enzimática. A atividade enzimática foi monitorada durante as alimentações e ao final do processo.

Em um processo SFS em batelada iniciando com $6 \%$ de celulose, contida no bagaço de cana, a quantidade de enzimas adicionada é referente a sua atividade (FPU) para a quantidade inicial de celulose $(60 \mathrm{~g} / \mathrm{L})$. Em um processo SFS em batelada alimentada, a quantidade de enzimas adicionada é referente a apenas $2 \%$ de celulose inicial e se a enzimas não perde atividade durante o processo, ela não precisa ser adicionada no sistema. Com isso, é possível se trabalhar com $1 / 3$ da quantidade de enzimas no processo em batelada alimentada em comparação com o processo me batelada. Outra problemática que instigou este trabalho foi a fato de que a agitação do processo em batelada é bem mais complicada no início devido à alta carga de bagaço de cana e esta dificuldade é reduzida quando se realiza o processo em batelada alimentada.

\subsection{Ensaios analíticos}

As quantificações dos açúcares, etanol e glicerol foram feitas por CLAE (Cromatografia líquida de alta eficiência) usando a coluna Shim-pack SCR 101-H (SHIMADZU, Kyoto/Japão) a $65{ }^{\circ} \mathrm{C}$ e ácido sulfúrico $5 \mathrm{mM}$ em água MiliQ (D7031, 
Barnstead EasyPure RF System - Iowa/EUA) como fase móvel, uma vazão de 0,6 mL/min e o índice de refração (RID-10A, SHIMADZU, Japão) como detector.

A determinação de celulose consumida foi de acordo com a NREL (National Renewable Energy Laboratoty) (Dowe e McMillan, 2001).

A determinação da biomassa celular para padronizar o inoculo foi realizada fazendo-se uma correlação entre massa seca em g/L e ABS em espectrofotômetro. Para o estudo do processo SFS, foi feita uma correlação entre a massa seca em $\mathrm{g} / \mathrm{L}$ e contagem de células viáveis em placa UFC/mL (Unidade Formadoras de Colônias), uma vez que, a bagaço de cana é um interferente na leitura de D.O (Densidade Ótica).

\section{RESULTADOS E DISCUSSÃO}

Na Figura 1 estão apresentados os resultados em do estudo cinético realizado em sistema batelada alimentada no ensaio CBA-B, bem como, o crescimento celular que foi feito durante o ensaio CBA-A.

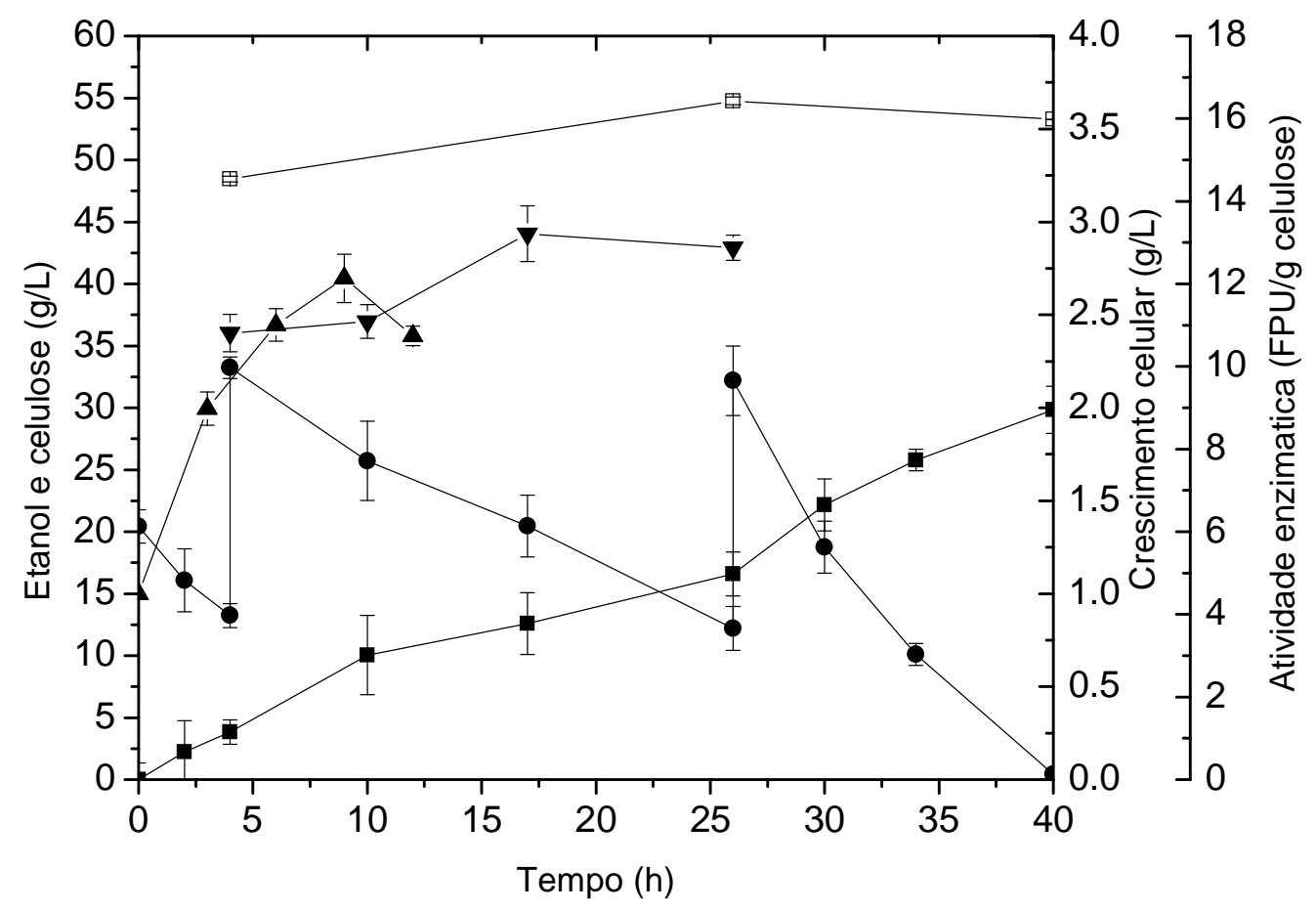

$(\boldsymbol{\bullet})$ Etanol produzido, $(\bullet)$ Perfil da celulose, $(\boldsymbol{\Delta})$ Crescimento celular na CBA-A, ( $\boldsymbol{\nabla})$ Crescimento celular na CBA-B depois da segunda alimentação, ( $\square$ ) Atividade enzimática. 
Figura 1: Perfil cinético do processo em batelada alimentada nos ensaios (CBA-A) e (CBAB).

Na Tabela 1 estão apresentados os resultados de produção de etanol e glicerol, a celulose residual do processo, o rendimento de etanol por grama de bagaço utilizado e o rendimento teórico do processo para os sistemas batelada e batelada alimentada partindo de 1,0 g/L de inoculo, 15 FPU/g de celulose e $6 \%$ de celulose total, contida no bagaço de cana, adicionada ao sistema.

Tabela 1 - Produção de etanol e glicerol, celulose residual, rendimento de etanol por grama de bagaço utilizado e rendimento teórico do processo.

\begin{tabular}{cccccc}
\hline $\begin{array}{c}\text { Condição } \\
\text { inicial }\end{array}$ & $\begin{array}{c}\text { Glicerol } \\
(\mathrm{g} / \mathrm{L})\end{array}$ & Etanol $(\mathrm{g} / \mathrm{L})$ & $\begin{array}{c}\text { Etanol } \\
(\mathrm{g} / \mathrm{g} \\
\text { bagaço })\end{array}$ & $\begin{array}{c}\text { Celulose } \\
\text { residual } \\
(\mathrm{g} / \mathrm{L})\end{array}$ & $\begin{array}{c}\text { Rendimento } \\
(\% \text { do Y } \\
\text { teórico })\end{array}$ \\
\hline Batelada $^{\mathrm{a}}$ & $1,21 \pm 0,1$ & $31,47 \pm 2,09$ & $0,34 \pm 0,03$ & $4,45 \pm 0,93$ & 92,58 \\
$\begin{array}{c}\text { Batelada } \\
\text { alimentada }\end{array}$ & $0,05 \pm 0,00$ & $29,83 \pm 0,96$ & $0,32 \pm 0,02$ & $0,44 \pm 0.12$ & 88,00
\end{tabular}

a-resultados de estudos anteriores com previsão para publicação neste mesmo evento em um trabalho intitulado: Estudo Cinético do Processo SFS em Biorreator para Produção de Etanol a Partir de Bagaço de Cana-de-açúcar.

Trabalhando no sistema batelada alimentada foi possível obter $29,83 \mathrm{~g} / \mathrm{L}$ de etanol e $88 \%$ de rendimento em comparação ao rendimento teórico (51\%) em 40 horas de processo, comparado às 24 horas no processo batelada e um rendimento um pouco maior, os resultados não parecem favorecer o processo, entretanto, a quantidade de enzima foram os $15 \mathrm{FPU} / \mathrm{g}$ celulose para $2 \%$ de celulose inicial e que se reutilizou nas outras duas alimentações de 2 e $2 \%$ de celulose contida no bagaço sem que houvesse alimentação de mais enzimas. Isso significa a utilização de apenas $1 / 3$ da quantidade de enzima que se utiliza no processo em batelada.

Outra vantagem encontrada no sistema em batelada alimentada foi a questão de uma melhor agitação do sistema o que favorecer a reologia do meio e que se espera que este fato reduza a demanda de potência do motor quando se pensa em ampliação de escala. Com esse estudo foi possível se trabalhar com um terço da quantidade de enzimas em comparação ao processo em batelada.

Como pode ser visto na Figura 1, 2,7 g/L de biomassa celular foi obtida em 10 horas de processo no estudo CBA-A, e depois um declínio. Este comportamento microbiano foi utilizado para estimar o final da fase exponencial que foi em 4 horas de processo. Portanto, foi em 4 horas que se fez a segunda alimentação de bagaço de cana no ensaio CBA-B, sendo a primeira alimentação os $2 \%$ de celulose inicial. Durante o estudo CBA-B o crescimento celular foi monitorado após a segunda alimentação e foi de $2,4 \mathrm{~g} / \mathrm{L}$ no início até se estabilizar em 2,9 g/L em 17 horas de processo. Neste trabalho o crescimento celular não foi monitorado após a terceira alimentação, pois os resultados não eram interessantes para a otimização do processo.

Os resultados cinéticos do estudo CBA-A foram utilizados para estimar o tempo da terceira alimentação de acordo com a taxa de consumo de celulose que estava em 1,47 g/L.h 
em 4 horas de processo. E neste caso não se levou em consideração a taxa de crescimento microbiano e produção de glicose, o que tornaria o cálculo mais complexo. Portanto, o tempo de alimentação ficou em 22 horas após a segunda alimentação, onde se viu que toda celulose estaria consumida, entretanto, quando se observa na Figura 1, pode-se notar que a celulose não foi totalmente consumida neste tempo. Neste caso o comportamento celular, uma nova fase lag, parece ter reduzido o consume de celulose, e em 26 horas de processo ainda restava $13 \mathrm{~g} / \mathrm{L}$ de celulose para ser consumida.

Após a terceira alimentação, a última etapa do processo, a celulose estava com $33 \mathrm{~g} / \mathrm{L}$ em concentração, mas como a quantidade de células estava maior e, ao que parece, se encontravam numa fase de maior consumo de substrato, a celulose foi rapidamente consumida em 14 horas. Ao que parece, a taxa de produção de glicose pelas enzimas celulases não limitaram o processo aqui estudado.

Se os resultados de crescimento de biomassa no ensaio CBA-B fossem utilizados para estimar a o final de fase Log a partir da segunda alimentação, este resultado seria utilizado para se fazer a terceira alimentação em um terceiro estudo cinético sem a necessidade de usar a taxa de consumo de celulose (g/L.h) como estimativa. Neste caso o tempo do final da segunda fase Log seria de 14 horas de processo total (Figura 1). Pela Figura 1, observa-se que alimentado $20 \mathrm{~g} / \mathrm{L}$ de celulose em 14 horas, a celulose aumentaria de $23 \mathrm{~g} / \mathrm{L}$ para $43 \mathrm{~g} / \mathrm{L}$ e a atividade enzimática que estava em $15 \mathrm{FPU} / \mathrm{g}$ de celulose cairia para 7,5 FPU/g de celulose o que poderia comprometer a agitação do sistema, bem como, o tempo de processo. Portanto, pode-se afirmar que o perfil visto na Figura 1 para o processo em batelada alimentada está perto do ideal e um terceiro estudo cinético não se faz necessário.

Ohgren et al (2007) produziu 21,4 g/L de etanol, 0,75 g etanol/grama de palha de milho e um rendimento de $81,5 \%$ em um reator de $15 \mathrm{~L} \mathrm{com} 5 \mathrm{~kg}$ de peso de biomassa. Desta forma, os resultados deste trabalho estão em concordância com a literatura.

\section{CONCLUSÃO}

Desta forma, foi possível reduzir custo com enzimas e demanda de potência do motor fazendo-se uso de uma estratégia de engenharia química, no caso o sistema em batelada alimentada, para se reduzir custo de processo obtendo-se excelentes rendimentos teóricos em etanol.

Um estudo com diferentes tipos de impelidores e frequência de agitação faz-se necessário quando se pensa em uma ampliação de escala deste processo. Desta forma, os resultados aqui apresentados são um ponto de partida para novos estudos do processo aqui explorado. 


\section{REFERÊNCIAS}

BASSO, L. C.; AMORIM, H. V.; OLIVEIRA, A. J.; LOPES, M. L. Yeast selection for fuel ethanol production in Brazil, FEMS Yeast Res, v. 8, p. 1155-1163, 2008.

CHANG, V.; HOLTZAPPLE, M. Fundamental factors affecting biomass enzymatic reactivity. Appl Biochem Biotechnol, v. 84-86, p. 5-37, 2000.

DOWE, N.; McMILLAN, J. SSF Experimental Protocol - Lignocellulosic Biomass Hydrolysis and Fermentatio. Laboratory Analytical Procedure (LAP), Technical Report, NREL, 2001. p. 19.

GHOSE, T. K. Measurement of cellulose activities. Pure Appl Chem. v. 59, p. 257-268, 1987.

GUO, G. L.; HSU, D. C.; CHEN, W. H.; CHEN, W.H.; HWANG, W. S. Characterization of enzymatic saccharification for acid-pretreated lignocellulosic materials with different lignin composition. Enzyme Microb Technol, v. 45, p. 80-87, 2009.

OHGREN, K.; BURA, R.; LESNICKI, G.; SADDLER, J.; ZACCHI, G. A comparison between simultaneous saccharification and fermentation and separate hydrolysis and fermentation using steam-pretreated corn stover. Process Biochemistry, v. 42, p. 834-839, 2007.

SCHELL, D. J.; FARMER, J.; NEWMAN, M.; MCMILLAN, J. D. Dilutesulfuric acid pretreatment of corn stover in pilot-scale reactor - investigation of yields, kinetics, and enzymatic digestibilities of solids. Appl Biochem Biotechnol, v. 105, p. 69-85, 2003.

SILVA, J. O.; COSTA, P. P.; RECHE, S. H. C. Manutenção de leveduras por congelamento a $-20{ }^{\circ}$ C. $R B A C$, v. 40, n. 1, p. 73-74, 2008.

SILVERSTEIN, R. A.; CHEN, Y.; SHARMA-SHIVAPPA, R. R.; BOYETTE, M. D. J. O. A comparison of chemical pretreatment methods for improving saccharification of cotton stalks. Bioresour Technol, v. 98, p. 3000-3011, 2007.

SUN, Y.; CHENG, J. Hydrolysis of lignocellulosic materials for ethanol production: a review. Bioresour Technol, v. 83, p. 1-11, 2002.

VASQUEZ, M. P.; SILVA, J. N. C.; SOUZA, M. B. Jr.; PEREIRA, N. Jr. Enzymatic hydrolysis optimization to ethanol production by simultaneous saccharification and fermentation. Appl Biochem Biotechnol, v. 136-140, p. 141-154, 2007.

YANG, P.; GUO, L.; CHENG, S.; LOU, N.; LIN, J. Recombinant multi-functional cellulase activity in submerged fermentation of lignocellulosic wastes, Renewable Energy. v. 36, p. 3268-3272, 2011.

ZHANG, K.; CHANG, J.; GUAN, Y.; CHEN, H.; YANG, Y.; JIANG, J. Lignocellulosic biomass gasification technology in China. Renewable Energy. v. 49, p. 175-184, 2013. 